\title{
"Thyroid nodular disease and PTEN mutation in a multicentre series of children with PTEN hamartoma tumor syndrome (PHTS)"
}

\author{
Gerdi Tuli $\mathbb{1}^{1,2} \cdot$ Jessica Munarin ${ }^{1,2} \cdot$ Alessandro Mussa $^{2} \cdot$ Diana Carli $^{2} \cdot$ Roberto Gastaldi $^{3}$ Paola Borgia ${ }^{3} \cdot$ \\ Maria Cristina Vigone ${ }^{4} \cdot$ Marco Abbate $^{4} \cdot$ Giovanni Battista Ferrero $^{2} \cdot$ Luisa De Sanctis $^{1,2}$
}

Received: 22 February 2021 / Accepted: 16 June 2021 / Published online: 28 June 2021

(C) The Author(s) 2021

\begin{abstract}
Purpose To report the incidence of $4-12 \%$ of differentiated thyroid cancer (DTC) and up to $50 \%$ of benign thyroid nodular disease and to describe nodular thyroid disease in a multicentre pediatric population with PTEN mutations. Methods: Retrospective data of pediatric patients with PTEN mutations collected from tertiary Departments of Pediatric Endocrinology of Turin, Milan and Genua, Italy, in the period 2010-2020.

Results Seventeen children with PTEN mutations were recruited in the study. Thyroid involvement was present in 12/17 (70.6\%) subjects, showing a multinodular struma in 6/17 (35.3\%), nodules with benign ultrasound features in 5/17 (29.4\%) and a follicular adenoma in 1/17 (6\%). No correlation was found between thyroid disease and gender, puberty, vascular manifestations, delayed development, or brain MRI abnormalities, while multiple lipomas were associated with thyroid disease $(p=0.03)$, as was macrocephaly. Standard Deviation (SD) score head circumference was $4.35 \pm 1.35 \mathrm{~cm}$ in subjects with thyroid disease, $3 \pm 0.43 \mathrm{~cm}(p=0.02)$ in the group without thyroid disease. Thyroid involvement was present in all subjects with mutations in exon $6(4 / 4)$ and exon $8(3 / 3)$ of the PTEN gene $(p=0.02)$.

Conclusion In the presented cohort, benign thyroid disorders were prevalent, with no evidence of DTC. A correlation was found between thyroid lesions and head circumference and the occurrence of multiple lipomas. Future studies in larger cohorts should assess whether risk stratification is needed when recommending surveillance strategies in children or young adolescents with PTEN hamartoma syndrome.
\end{abstract}

Keywords PTEN mutation $\cdot$ Pediatric age $\cdot$ Thyroid nodule $\cdot$ Differentiated thyroid cancer

\section{Introduction}

The phosphatase and tensin homolog (PTEN) gene, located on 10q23 chromosome band, is a tumor suppressor gene with a fundamental role in the molecular pathways that mediate cell proliferation, migration, and apoptosis. Germline PTEN mutations, inherited with an autosomal dominant

Gerdi Tuli

gerdi.tuli@unito.it

1 Department of Pediatric Endocrinology, Regina Margherita Children Hospital, Turin, Italy

2 Department of Public Health and Pediatrics, University of Turin, Turin, Italy

3 Department of Paediatrics Gaslini Hospital, Genova, Italy

4 Department of Pediatrics, IRCCS San Raffaele Scientific Institute, Milan, Italy mechanism, have been related with the etiology of "PTEN hamartoma tumor syndromes" (PHTS), which include Cowden syndrome (CS, OMIM 158350), Bannayan-RileyRuvalcaba syndrome (BRRS, OMIM 153480) and PTENrelated proteus syndrome (PS, OMIM 176920) [1-5].

BRRS is considered the PHTS form of pediatric age and is characterized by macrocephaly, benign hamartomas, pigmented glans macules, lipomas, hemangiomas, and developmental delay or the presence of an autistic trait. Other phenotypic features of BRRS disorder include prenatal or postnatal onset of high-arched palate overgrowth, macrosomia, hypotonia, joint hyperextensibility, downward slanting palpebral fissures, frontal bossing, hypoglycemia, seizures, and café au lait spots. These phenotypic features are highly variable, although they seem to cluster within the same family [1-5].

Patients with PTEN mutations are at higher risk for malignancies of the breast, endometrium, colon, kidney and thyroid. Differentiated thyroid cancer (DTC) is one of the 
most common types of cancer, with a lifetime risk of 35$38 \%$ [6]; the increased risk is also present in pediatric age. Several authors report an incidence of $4-12 \%$ of DTC in the pediatric population affected by PTEN mutations, mainly follicular, and up to $50 \%$ of benign nodular thyroid disease [6]. Actually, there are no data on the behavior of such tumors but a surveillance programme is absolutely needed in these patients. Most guidelines agree on the annual surveillance program from the age of $7-10$, preferring to postpone the age of initiation of screening to 10 years to avoid overdiagnosis and family anxiety [6-9].

The rationale of the surveillance program is to detect thyroid cancer at an early stage, to prevent surgical complications such as hypoparathyroidism and recurrent nerve injury, and to avoid high doses of radioiodine treatment when needed.

In this paper, we describe nodular thyroid disease in a multicentre cohort of 17 patients with PTEN mutation and discuss the DTC surveillance program in childhood.

\section{Materials and methods}

Retrospective data were collected from all pediatric patients affected by PTEN mutation who referred to the Departments of Pediatric Endocrinology of Regina Margherita Children's Hospital of Turin, San Raffaele Hospital of Milan and Giannina Gaslini Hospital of Genua between the years 2010 and 2020.

Patients data were extracted from the electronic medical records, such as clinical features of PHTS, anthropometric data, type of PTEN gene mutation, thyroid hormone profile, thyroid ultrasound features, and cytological [based on the Italian Consensus Statement of 2014 (10)] and histological data.

Statistical analysis and graphs were performed using Graphpad 7 software (GraphPad Software, La Jolla, CA, USA) by the chi-quadrate test to compare differences between groups. The calculations were considered statistically significant when the $P$ value was less than 0.05 .

\section{Results}

Seventeen children and adolescents ( 9 males and 8 females, age at diagnosis $7.53 \pm 3.81$ years old) with PTEN mutation were recruited; their clinical data are presented in Table 1. The follow up period was $4.71 \pm 0.88$ years

PTEN mutation involved exon 1 in 4/17 subjects (23.5\%), exon 5 in $1 / 17$ (6\%), exon 6 in $4 / 17(23.5 \%)$, exon 7 in $4 / 17$ (23.5\%) and exon 8 in $3 / 17$ (17.5\%); in $1 / 17(6 \%)$ a deletion of chromosome 10 was detected, which included the PTEN gene. Familiarity for PTEN mutations was present in $10 / 17(58.8 \%)$, while a de novo mutation was detected in the remaining 7/10 (41.2\%) cases.

Anthropometric data showed macrocephaly in all cases (head circumference SDS $3.89 \pm 1.52 \mathrm{~cm}$ ), with height and BMI adequate for age and gender.

Considering the other abnormalities associated with PTEN mutation, multiple lipomas were present in 10/17 $(58.8 \%)$. Vascular manifestations were found in $5 / 17$ $(29.4 \%)$, mostly represented by angiomas. Delayed development was present in nearly all children and adolescents (14/17, 82.4\%), while brain MRI showed abnormalities, i.e., ventricular dilatation or periventricular leucomalacia, in 5/ $17(29.4 \%)$.

Thyroid gland involvement, as shown in Table 2, was present in $12 / 17(70.6 \%)$ subjects and mostly showed multinodular struma $(6 / 17,35.3 \%)$ or nodules with benign ultrasound features $(5 / 17,29.4 \%)$; follicular adenoma was present in $1 / 17(6 \%)$. The mean age at diagnosis of thyroid disease was $9.43 \pm 3.2$ years old, with an age ranging $2.52 \pm 1.56$ years after the diagnosis of PHTS. There was no correlation between thyroid disease and gender or puberty, or with vascular manifestations, delayed development and brain MRI abnormalities, while multiple lipomas were associated with thyroid disease $(p=0.03)$, as well as macrocephaly. SDS head circumference was $4.35 \pm 1.35 \mathrm{~cm}$ in subjects with thyroid disease, $3 \pm 0.43 \mathrm{~cm}(p=0.02)$ in the group without thyroid disease. Thyroid involvement was present in all subjects with mutations in exon $6(4 / 4)$ and exon $8(3 / 3)$ of the PTEN gene $(p=0.02)$. Changes in exon 1 showed thyroid disease in $50 \%$ of cases (2/4), while the majority of patients with exon 7 variations showed no thyroid manifestations (1/4 displayed multinodular struma).

Thyroid hormone profile was normal and anti-thyroid antibodies were negative in all subjects. Total thyroidectomy was performed in 4 of 17 patients $(23.5 \%)$ for a major multinodular struma with nodules $>1 \mathrm{~cm} \mathrm{(2/17)} \mathrm{or} \mathrm{for}$ cytological indications (1 with TIR3A and 1 with TIR5). Three subjects showed TIR2 cytology category after FNAB. Adenomatous nodules were present in 3 cases, while follicular adenoma was detected in 1 subject.

\section{Discussion}

Thyroid cancer surveillance programs are recommended in patients with PTEN mutations due to the well-known increased risk of developing thyroid cancer. Recent literature data report a 4-12\% prevalence of thyroid cancer in subjects with PTEN mutations, consistent with a low-cost, non-invasive screening program. To avoid delayed diagnosis and prevent surgical complications, as well as high doses of radioiodine treatment for metastatic thyroid cancer, ultrasound surveillance is indicated [6-9]. 


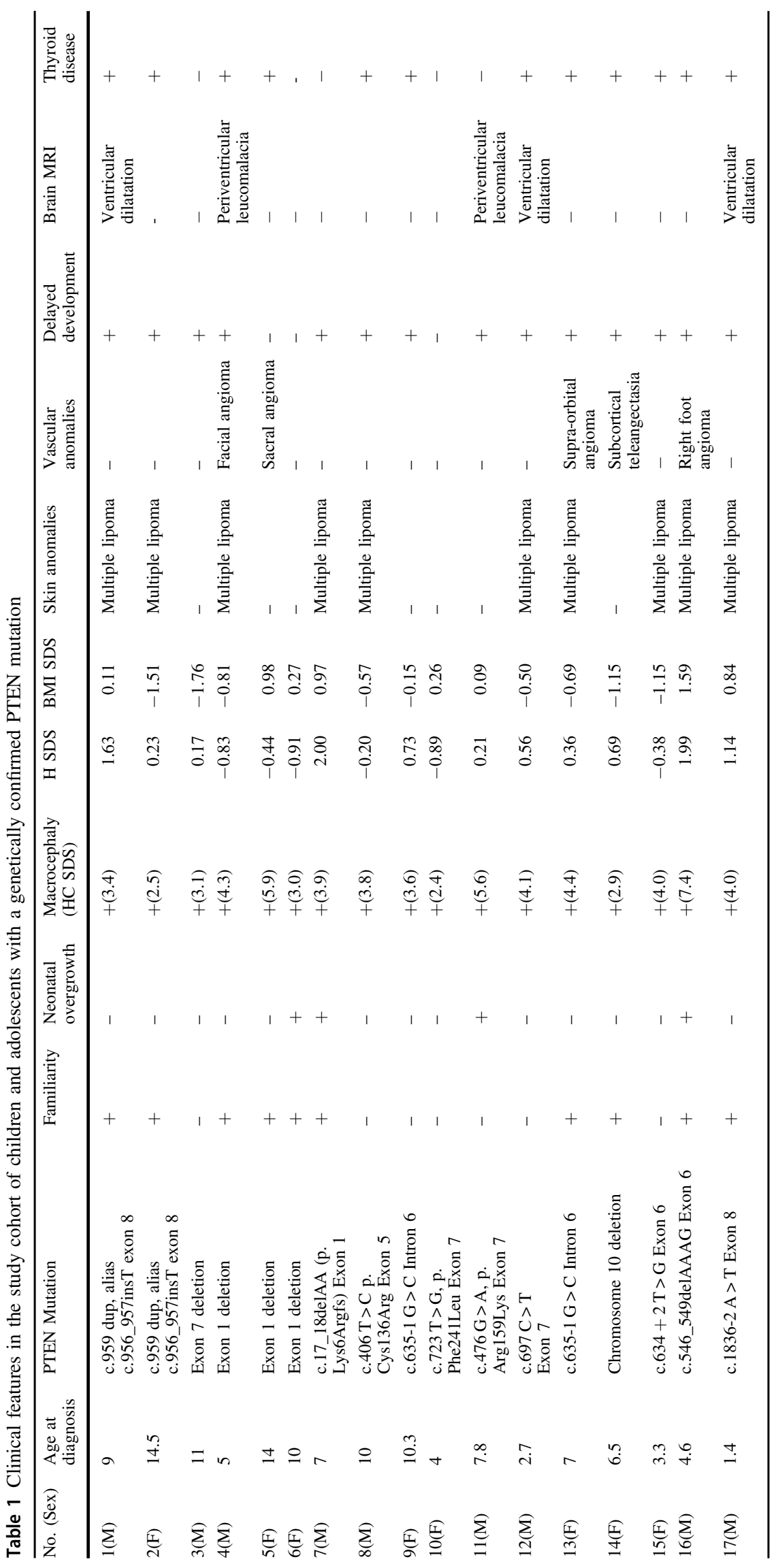




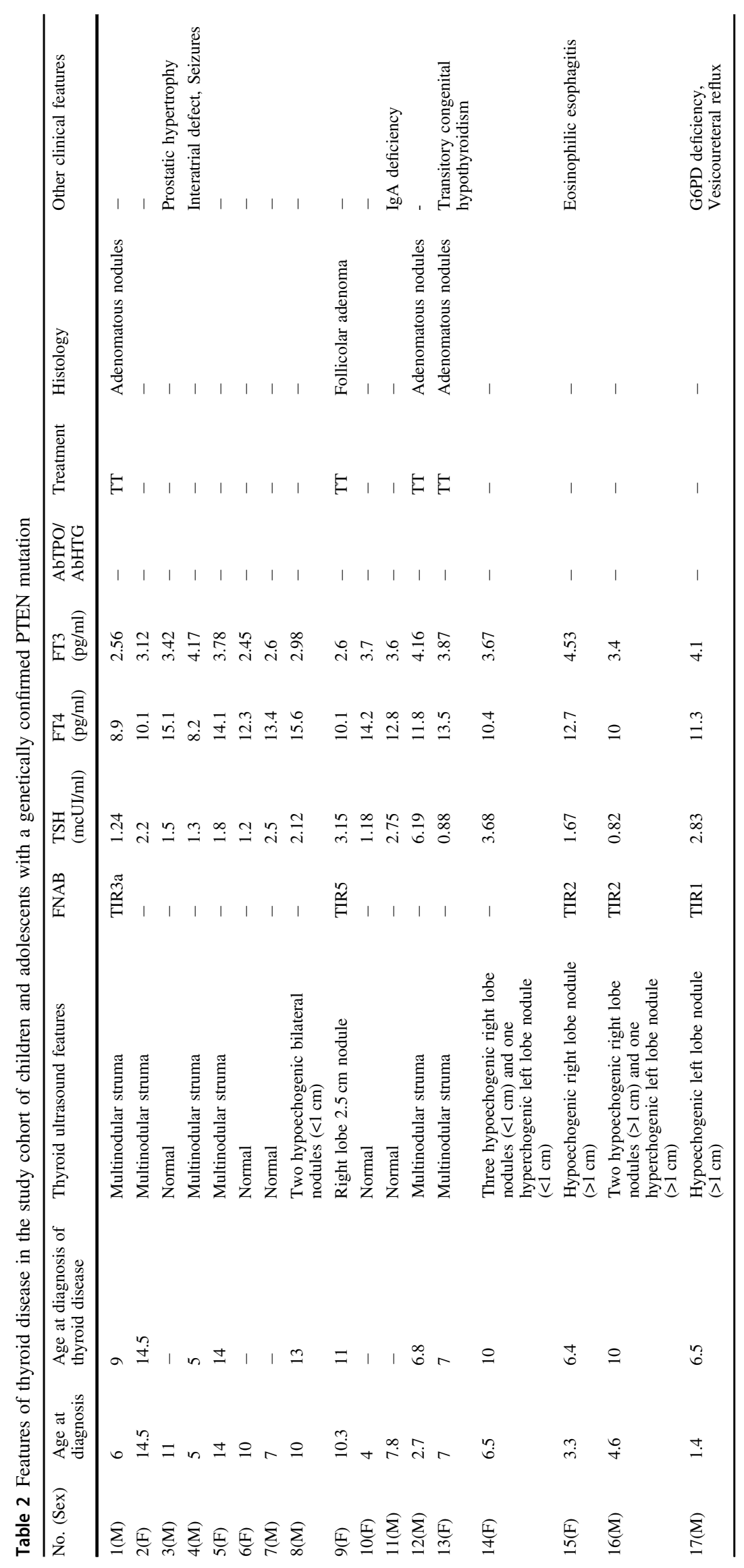


Most authors have suggested that thyroid disease surveillance should begin at a very young age (7-10 years old), with an annual or biennial ultrasound analysis. In our cohort, nearly half of the subjects with nodular thyroid disease $(5 / 12,41.7 \%)$ were less than 7 years old, unlike what has been reported by other authors who point to thyroid nodules as a rare finding in young children with PTEN mutations [6, 7]. This finding might be explained by the fact that in most cases of our cohort the PTEN gene analysis was performed at a young age for the presence of macrocephaly. However, the natural history of nodular disease in subjects with mutated PTEN does not appear to be very aggressive, as confirmed by the lack of evidence of DTC in the mean follow-up period of nearly 5 years in our cohort. This is encouraging about the predominance of the benign nodular disease in children with PTEN mutations and therefore, as suggested in recent recommendations, the appropriate age for initiation of DTC surveillance could be 10 years old [6].

In our cohort, $70.6 \%$ of subjects had nodular thyroid disease with a $47 \%$ rate of nodules $>1 \mathrm{~cm}$, in agreement with literature data [6-9]. In our cohort, no age or gender differences were observed between subjects without thyroid disease and those with thyroid nodules, as well as between subjects with nodules $>1 \mathrm{~cm}$ and $<1 \mathrm{~cm}$. Smith et al. in a larger cohort of 64 pediatric patients showed a significant difference in timing of diagnosis between males (10-15 years) and females (8-13 years), which corresponds with the pubertal period for both genders.

Among the various clinical features of PHTS, a possible correlation between nodular thyroid disease and head circumference and the presence of multiple lipoma emerged in the analyzed cohort. Thus, a head circumference $>4$ SDS might be associated with a higher risk of thyroid nodules, such as the presence of multiple lipomas with a greater likehood of nodular thyroid disease. Undoubtedly, these two possible associations, not previously reported by other authors, need to be confirmed in larger cohorts.

A possible correlation between genotype and thyroid disease was also observed in the cohort presented here, as all the subjects harboring mutations in exons 6 and 8 of the gene showed thyroid nodules. However, this finding also needs to be confirmed in larger cohorts, as no genotypephenotype correlation between PTEN mutations and thyroid disease has been reported so far [6-9].

Among nodular thyroid disease, the presence of multinodular struma was the most common finding (35.3\%), followed by benign nodular disease $(29.4 \%)$. No malignant nodules were detected in our cohort and fine needle biopsy (FNAB) was performed only in case of nodules $>1 \mathrm{~cm}$ or when ultrasound features were highly suspicious of malignancy. Total thyroidectomy was chosen in all cases of surgery; cytological analysis showed adenomatous nodules in 3 cases and follicular adenoma in 1 female subject. We agree with the recommendation of other groups that in these patients, when surgery is performed, the total thyroidectomy approach is the most advisable option in pediatric age [6-9].

All the subjects we collected had a normal thyroid hormone profile and none showed anti-peroxidase or antithyroglobulin antibodies, as reported by many authors [6-9].

We are aware that the limitations of this study are the retrospective nature of the data collection and the small size of the cohort, which limit the strength of the statistical analysis.

Therefore, the association of the severity of macrocephaly and multiple lipomas with nodular thyroid disease needs to be confirmed in larger cohorts, as well as the involvement of exon 6 and 8, which appears to increase the risk of nodular disease.

In conclusion, nodular thyroid disease is often the first serious complication in children with PTEN mutations, who have a higher risk of thyroid cancer. Therefore, an ultrasound surveillance program is recommended in all patients from infancy to avoid delayed diagnosis. The frequency of ultrasound screening should be decided on the basis of the first evaluation after confirmation of the PTEN mutation, but annual monitoring may be proposed, especially if severe macrocephaly is present. The proposed interval has to be modified and adapted when suspicious features of malignancy are present. In these cases, a fine needle biopsy is recommended and, if surgery is required, total thyroidectomy should be preferred.

Declaration section The manuscript has not been submitted to more than one journal for simultaneous consideration and has not been published previously. No data have been fabricated or manipulated (including images) to support our conclusions. No data, text, or theories by others are presented as if they were the author's own. The Ethical Committee of our Institution approved this study and parents have given their written informed consent

Author contributions Consent to submit has been received explicitly from all co-authors. All authors whose names appear on the submission have contributed sufficiently to the scientific work and therefore share collective responsibility and accountability for the results. G.T., A.M., M.C.V., R.G., G.B.F. and L.d.S. have contributed to the study design, statistical analysis and manuscript writing; J.M., D.C., P.B., M.A., to data collection and checking references.

Funding Open access funding provided by Universit Ã degli Studi di Torino within the CRUI-CARE Agreement.

\section{Compliance with ethical standards}

Conflict of interest The authors declare no competing interests.

Ethical approval All procedures performed in studies involving human participants were in accordance with the ethical standards of the institutional and/or national research committee and with the 1964 Helsinki declaration and its later amendments or comparable ethical standards. This article does not contain any studies with animals performed by any of the authors 
Informed consent Informed consent was obtained from all parents of the individual participants included in the study.

Publisher's note Springer Nature remains neutral with regard to jurisdictional claims in published maps and institutional affiliations.

Open Access This article is licensed under a Creative Commons Attribution 4.0 International License, which permits use, sharing, adaptation, distribution and reproduction in any medium or format, as long as you give appropriate credit to the original author(s) and the source, provide a link to the Creative Commons license, and indicate if changes were made. The images or other third party material in this article are included in the article's Creative Commons license, unless indicated otherwise in a credit line to the material. If material is not included in the article's Creative Commons license and your intended use is not permitted by statutory regulation or exceeds the permitted use, you will need to obtain permission directly from the copyright holder. To view a copy of this license, visit http://creativecommons. org/licenses/by/4.0/.

\section{References}

1. Ciaccio C., Saletti V., D’Arrigo S., Esposito S., Alfei E., Moroni I., Tonduti D., Chiapparini L., Pantaleoni C., Milani D. Clinical spectrum of PTEN mutation in pediatric patients. A bicenter experience. Eur. J. Med. Genet. (2019) https://doi.org/10.1016/j. ejmg.2018.12.001

2. G.M. Blumenthal, P.A. Dennis, PTEN hamartoma tumor syndromes. Eur. J. Hum. Genet. 16, 1289-1300 (2008)

3. R.J. Gorlin, M.M. Cohen Jr, L.M. Condon, B.A. Burke, Bannayan-Riley-Ruvalcaba syndrome. Am. J. Med. Genet. 44, 307-314 (1992)

4. D.J. Marsh, V. Coulon, K.L. Lunetta, P. Rocca-Serra, P.L. Dahia, Z. Zheng, D. Liaw, S. Caron, B. Duboué, A.Y. Lin, A.L. Richardson, J.M. Bonnetblanc, J.M. Bressieux, A. CabarrotMoreau, A. Chompret, L. Demange, R.A. Eeles, A.M. Yahanda, E.R. Fearon, J.P. Fricker, R.J. Gorlin, S.V. Hodgson, S. Huson, D.
Lacombe, C. Eng et al. Mutation spectrum and genotypephenotype analyses in Cowden disease and Bannayan-Zonana syndrome, two hamartoma syndromes with germline PTEN mutation. Hum. Mol. Genet. 7, 507-515 (1998)

5. D.J. Marsh, V. Coulon, K.L. Lunetta, P. Rocca-Serra, P.L.M. Dahia, Z. Zheng, D. Liaw, S. Caron, B. Duboué, A.Y. Lin, A.L. Richardson, J.-M. Bonnetblanc, J.-M. Bressieux, A. Cabarrot-Moreau, A. Chompret, L. Demange, R.A. Eeles, A.M. Yahanda, E.R. Fearon, J.-P. Fricker, R.J. Gorlin, S.V. Hodgson, S. Huson, D. Lacombe, F. LePrat, S. Odent, C. Toulouse, O.I. Olopade, H. Sobol, S. Tishler, C.G. Woods, B.G. Robinson, H. C. Weber, R. Parsons, M. Peacocke, M. Longy, C. Eng, Mutation spectrum and genotype-phenotype analyses in cowden disease and Bannayan-Zonana Syndrome, two Hamartoma syndromes with germline PTEN mutation. Hum. Mol. Genet. 7, 507-515 (1998)

6. L.A. Jonker, C.A. Lebbink, M.C.J. Jongmans, T.P. Links, N. Hoogerbrugge, A.S.P. van Trotsenburg, H.M. van Santen, Reccomendations on surveillance for differentiated thyroid carcinoma in children with PTEN hamartoma tumor syndrome. Eur. Thyroid. J. 9, 234-242 (2020)

7. Smith J.R., Liu E., Church A.J., Asch E., Cherella C., Srivastava S., Kamihara J., Wassner A.J. Natural history of thyroid disease in children with PTEN hamartoma tumor syndrome. J. Clin. Endocrinol. Metab. (2020) https://doi.org/10.1210/clinem/dgaa944

8. M. Plamper, F. Schreiner, B. Gohlke, J. Kionke, E. Korsch, J. Kirkpatrick, M. Born, S. Aretz, J. Woelfle, Thyroid disease in children and adolescents with PTEN hamartoma tumor syndrome (PHTS). Eur. J. Pediatr. 177, 429-435 (2018)

9. K.A.P. Schultz, S.P. Rednam, J. Kamihara, L. Doros, M.I. Achatz, J.D. Wasserman, L.R. Diller, L. Brugieres, H. Druker, K.A. Schneider, R.B. McGee, W.D. Foulkes, PTEN, DICER1, FH and their associated tumor susceptibility syndromes: clinical features, genetics and surveillance recommednations in childhood. Clin. Cancer Res. 23, e76-e82 (2017)

10. F. Nardi, F. Basolo, A. Crescenzi, G. Fadda, A. Frasoldati, F. Orlandi, L. Palombini, E. Papini, M. Zini, A. Pontecorvi, P. Vitti, Italian consensus for the classification and reporting of thyroid cytology. J. Endocrinol. Invest. 37, 593-599 (2014) 